\title{
Más allá de lo erótico. La sumisión sexual en el personaje de Samantha
}

\author{
Inés Bejar Gallardo \\ Universidad de Sevilla \\ pepeniak@gmail.com \\ https://dx.doi.org.10.12795/futhark.2017.il2.02
}

Fecha de recepción: II.5.2017

Fecha de aceptación: 15.06.2017

Resumen: Este trabajo aborda la sumisión sexual en el personaje de Samantha. Para ello se realiza un estudio de caso de la novela Jax Warrior Lover. En el presente análisis se esbozan algunas de las líneas vectoriales que separan las fronteras genéricas y estéticas entre el erotismo y lo pornográfico. El artículo de investigación se mueve en formatos masivos de la cultura popular mediática. Con especial detenimiento en los programas de telerrealidad y las redes sociales. La representación en medios audiovisuales y la literatura académica que versa sobre esta realidad compleja son la fuente principal de trabajo de la investigación.

Palabras clave: erótico, mujer, sexo, sumisión, violencia.

\section{Beyond the erotic. Sexual submission in Samantha's character}

\begin{abstract}
This work is about Samantha's character sexual submission. A case study of Jax Warrior Lover novel will be made. In this analysis outlines vectorial lines that separate the generic and aesthetic boundaries between eroticism and pornography. Article research uses in massive formats of popular media culture. With special attention to reality shows and social networks. Representation in audiovisual media and academic literature that deals with this complex reality will be the main source of research work.
\end{abstract}

Key words: erotic, women, sex, submission, violence.

Sumario: Introducción. I. La sumisión sexual en el personaje de Samantha. 


\section{Introducción}

En un futuro catastrófico, la autora Inka Loreen Minden (2013) nos presenta un planeta destrozado y una sociedad dividida entre los oprimidos y los que oprimen'. Este panorama en principio no tiene que nada que no se haya visto con anterioridad en otras obras ${ }^{2}$, ya que casi siempre se describe el futuro como un mundo sin recursos y con sociedades que llegan al límite de lo moral y lo racional. Pero en este caso, esa degeneración parece imposible que pueda darse lugar en la sociedad occidental actual ${ }^{3}$. Aborda un tema tabú ${ }^{4}$ que se supone no tiene voz y menos entre el sexo femenino $0^{5}$, pero sí la tiene. Es un mundo en el que las mujeres quieren sentirse sometidas hasta ese punto, alejándose así de la realidad, donde son

\footnotetext{
${ }^{1}$ En este sentido, estas sociedades futuristas reflejan de un modo otro, el presente en el sentido más extremo. dramática lo que ocurre hoy en día en muchas partes del mundo, pues las políticas agresivas y los gobiernos totalitarios son las causantes de la desvalorización de lo humano.

2 En obras literarias como Un mundo feliz (1932) de Aldou Huxeley, (1953) Farenheit 45I de Rey Bradbury, incluso El Señor de las moscas (1954) de William Golding, El cuento de la criada (1985) de Margaret Atwood o La carretera (2006) de Cormac McCarthy, podemos ver ya estos ambientes futuristas, planteados como dramáticos y trágicos, con la decadencia de la mentalidad del hombre en situaciones extremas y la devastación del planeta.

${ }^{3}$ La violación sexual, a pesar de ser un hecho que está a la orden del día en todo el mundo, está penada por ley en la mayoría de las sociedades y se castiga a quien comete este tipo de acciones. Preciado (2007) escribe en su texto sobre la violación y la pornografía: "apunta bajo el eslogan de Robín Morgan "la pornografía es la teoría, la violación la práctica", condenan la representación de la sexualidad femenina llevada a cabo por los medios de comunicación como una forma de promoción de la violencia de género, de la sumisión sexual y política de las mujeres y abogan por la abolición total de la pornografía y la prostitución" (Preciado 2007: 2).

${ }^{4}$ Meza Villalba (2018) apunta que: "cuando se habla de sexo es natural que la gente se sienta incomoda por ser un tema del cual no es fácil de hablar en cualquier momento o espacio. Todo esto pasa por el pensamiento moral que se ha instaurado de que la sexualidad es prohibido [sic] o que su disfrute debe ser discreto a tal punto de ser impuro. La sociedad occidental actual, ha ido convirtiendo al sexo en un tabú" (Meza 2018: 2). En este sentido, el deseo de ser sumisas y dominadas es un acto con el que se lleva luchando muchos años, pero hay mujeres que desean esa dominación. Como anota Calvo Shadid (20I3): "El ámbito en el que se delimita el tabú es muy amplio; opera en lugares, acciones, en la lengua, en personas y en situaciones. De tal modo lo expresa Guérios (1956:7): 'Así, existen objetos tabú [sic] que no deben ser tocados; lugares tabú que no deben ser pisados o a los que no se debe acercar; acciones tabú que no deben ser practicadas, y palabras tabú que no deben ser proferidas. Además de esto, hay personas tabú [sic] y situaciones o estados tabú" (Calvo 2013:123).

${ }^{5}$ Tradicionalmente hablar de sexo entre mujeres quedaba en el marco del círculo más íntimo, en el caso de que se diera, pues la mujer ha sido hasta hace relativamente poco el objeto de deseo, y no el participante. Las mujeres siempre han sido más cautas al hablar de sexo, pues nunca ha estado bien considerado. Morales (2005) dice que: "los hombres utilizan más tabúes que las mujeres y consecuentemente menos tecnicismos y eufemismos, hoy en día ha cambiado esta situación, pero sigue siendo común que las mujeres no expresen sus deseos sexuales abiertamente” (Morales 2005: 14).
} 
ellas las que dominan su vida y deciden su destino ${ }^{6}$. El hecho de que Samantha, la protagonista, sea médica muestra esa mujer que se aleja de los estereotipos ${ }^{7}$. Una mujer con estudios superiores, con una profesión que le permite ser independiente, que controla su vida, puede sentir el deseo de querer ser dominada.

Va más allá de lo erótico ${ }^{8}$ la autora pone de manifiesto los deseos sexuales más inconfesables, mostrando situaciones límites. Si observamos la opinión de sus lectores, el éxito ${ }^{9}$ de sus novelas se debe a que traslada esos deseos íntimos, se atreve a escribir sobre ellos y compartirlos con todo el mundo ${ }^{10}$. Es por lo que sus libros no se venden en papel, solo en formato electrónico, porque son temas que entran dentro de lo prohibido, pero no así de lo obsceno o pornográfico".

\footnotetext{
${ }^{6}$ En este sentido Castellanos apunta (2006: 55): "Encontramos una extensa literatura... que muestran las mujeres humilladas o agredidas en relación con actos sexuales" Esta literatura suele ser reflejo de la realidad histórica del hombre y la mujer. Históricamente ha predominado la literatura masculina, por lo que el hecho de que una mujer escriba sobre estos temas sitúa a la misma en un lugar de igualdad en cuanto a ideas, sentimientos y creación literaria.

7 Bravo y Moreno (2007) definen lo que suponen los estereotipos: "los estereotipos constituyen... herramientas socioculturales sobre las que se asientan normas de funcionamiento social a la vez que sirven de referente para estructurar la identidad de los sujetos" (Bravo/ Moreno 2007: 239). Por lo que las personas asocian un tipo de actitud y manera de pensar, en este caso vemos a la protagonista como ejemplo de personaje no estereotipado.

8 Llorente (2013: 360s.) nos define erotismo como "evocación o sublimación, consciente o inconsciente, del hecho sexual". Y la pornografía como "exhibición de actos sexuales explícitos". A partir de estas definiciones, Llorente afirma que: "existe un límite operativo, a partir del cual la atracción de lo que se considera comúnmente como erotismo empieza a convertirse en el rechazo de lo [sic] también comúnmente entendido por pornografía u obscenidad... lo erótico se presenta siempre como algo único al concepto de limite".

${ }^{9}$ En el siguiente enlace se encuentran las opiniones de algunos lectores donde se han podido ver las valoraciones, en su mayoría, positivas de la mayoría de ellos: https://www.goodreads.com/book/show/ 18305। 34-jax.

${ }^{10}$ El siguiente enlace es de la página web de la autora, donde se pueden ver todas sus novelas que incluyen una sinopsis y se puede ver la temática general de sus obras, http://www.inka-loreen-minden.de

1 Castellanos (2006: 54) nos define lo que es erótico diferenciándolo de lo pornográfico y lo obsceno: "Lo erótico, por otro lado, debe diferenciarse de lo obsceno, mencionado a menudo en los juicios contra la pornografía. Asimismo, la crítica feminista a la pornografía y la persecución del Estado liberal a la obscenidad son dos cosas totalmente distintas. El concepto de obscenidad nos remite a la representación de la sexualidad que una sociedad juzga como ofensiva, y está ligado a la moralidad y a las normas sociales. La pornografía, en cambio, está a menudo ligada a la representación de la violencia. Precisamente una de las diferencias entre pornografía y erotismo es la ausencia de coerción o de violencia en las representaciones eróticas".
} 


\section{La sumisión sexual en el personaje de Samantha}

Samantha es el personaje que nos cuenta su historia, en la que sufre ya que es una esclava y su castigo es esperar a ser violada por un guerrero. En las primeras líneas del texto nos presenta esta situación límite, pero dentro de esta ya nos muestra el erotismo que va a tener el relato, "Wir Sklaven tragen bloß einen blutroten Stringtanga, die Frauen zusätzlich ein Brustband in derselben Farbe. Es verhüllt kaum unsere Nippel. Niemals zuvor bin ich mir so nackt vorgekommen" (Minden 2013: 3) ${ }^{12}$.

No se detiene en la descripción de los ambientes donde ocurren los hechos. Nos cuenta lo necesario para hacernos una idea del momento y el espacio en que es narrada la historia, porque lo que importa son los personajes y esos hechos. Pero sí cuida el sentido que tienen esos ambientes en la novela. Primero, un lugar destinado para la diversión del pueblo, para el espectáculo, donde éste observa y juzga. La autora justifica así, esa necesidad de contar, de que todo el mundo lo vea, de ser observada en lo más íntimo de su mente. Un deseo que no todos los que lo tienen se atreven a expresar. $Y$ el segundo, un entorno claramente propicio para el sexo $^{13}$, como es la habitación de un lujoso hotel, donde el público sigue observando. En este segundo lugar, describe la situación de manera que el tema de fondo llega a olvidarse debido a la tensión sexual ${ }^{14}$ más evidente, que va entrelazando con las acciones y reacciones de miedo y posesión de los protagonistas. Todo ello, como se ha mencionado, con espectadores que lo ven todo, una representación de los Realitys Shows"15, "As New York Times writer

\footnotetext{
12 Todas las citas de este libro serán de la misma edición. Inka L. Minden (20I3) Jax Warrior Lover.

${ }^{13}$ Es evidente el atractivo sexual de estos escenarios, pues son modelos de escenas románticas, eróticas y sexuales en todo tipo de relatos. Un previo ejemplo de ello lo vemos en la popular novela libro Fifty shades of Grey (20II) de Erika Leonard Mitchell. Esta novela erótica alcanzó un gran éxito, obteniendo muchos seguidores y detractores. La primera cita sexual que tiene los protagonistas en la habitación de un hotel. Puede ser esta la primera novela que abrió las puertas al público de este tipo de erotismo femenino hoy en día En plataformas virtuales como goodreads.es se puede ver la opinión de los lectores y el éxito de esta novela: https://www.goodreads.com/book/show/l08/8853-fifty-shades-of-grey? fromsearch=true y https://www.goodreads.com/award/show/405-all-about-romance-aar-annual-readerpoll.

${ }^{14}$ El hecho de mantener la tensión en cualquier aspecto hace que cobre interés, este Es un recurso ha sido utilizado siempre con éxito de todo para mantener la atención de los lectores.

${ }^{15}$ Esto ya lo anunciaba George Orwell en su libro 1984 (1949). Los programas de televisión Reality Shows, son los llamados programas de telerrealidad en español. El primer programa que existió en este sentido fue the Big Brother en Holanda. Surgió como experimento sociológico, donde el fin es observar el comportamiento y la convivencia de personas encerradas en un determinado lugar. Donde los concursantes son observados 24 horas durante un determinado periodo de tiempo. Tuvo un éxito mundial y hoy en día sigue emitiéndose en muchos países, en algunos de manera intermitente. Hill (2002) nos habla del atractivo de estos programas de televisión: "but perhaps the most striking reasons for watching BB are that everybody else is watching and talking about it, and everybody else is forming 
Alessandra Stanley (2002, p. Al) puts it, "humiliation is the unifying principle behind a successful reality show" (en Mendible 2004: 2). Por lo que la protagonista de este relato sufre este tipo de sensación constante. Asimismo, trata la posibilidad que hay en sentir miedo y vergüenza y a la vez excitarse sexualmente, y cómo esos sentimientos pueden darse lugar en una situación extrema.

Los personajes, que se presentan con el desarrollo de la narración, tienen una descripción física más detallada, son cánones de belleza ${ }^{16}$, sus rasgos y características físicas muestran la imagen de la sexualidad más atractiva.

"Body image is a construct determined by a series of individual and sociocultural factors that intervenes decisively in several aspects of our lives in Western society Amongst the broader sociocultural determinants of body image development are ideal body representations transmitted through various socialisation agents such as the mass media, which clearly stands out as spokespeople for these cultural values" (Sepúlveda y Calado 2012: 47).

Por un lado, los guerreros, entre ellos Jax, se describen como “... denn die Krieger sin alle groß, mit einem breiten Rücken un Muskeln wie Stahl. Sein silberfarbenes fast weißes Haar leuchtet, als das Licht der Avheinwefer auf ihn fällt" (Minden 5), una descripción de atractivo físico para la mayoría de las personas. Por otro lado, "la subordinación de la mujer al hombre se erotiza" (Castellanos 2006: 55). El personaje femenino, se ve a sí mismo como alguien poco atractivo físicamente, pues se describe como "Zumindest entspreche ich nicht dem gängigen Schönheitsideal. Habe keinen Korret-Maß-Körper. Meine Bruste sin zu groß, mein Hintern zu rund und ich wirke wenig burschikos" (Minden 5). Pero es precisamente este físico el que ha pasado a ser un modelo de cuerpo atractivo sexual en los tiempos actuales por lo que podemos ver los medios de comunicación, ${ }^{17}$ como ya

judgments on the contestants and how they act up for the cameras" (Hill 2002: 324). Este formado ha derivado en multitud de tipos de programas de este tipo, donde los escenarios y los personajes son diferentes pero el fin es el mismo. Hoy en día es la mera observación de los demás en situaciones de todo tipo. Asimismo, Hill se pregunta sobre la telerrealidad: "Big Brother is a new format in factual entertainment that has been subject to much speculation in the press, on TV, the internet, and by the public. As the Big Brother (BB) format was quickly adopted by different countries, this speculation focused primarily on the negative: was Big Brother voyeuristic?" (Hill 2002: 323).

16 Sepúlveda y Calado (2012: 47) continúan en este sentido: "The mass media serves as a mediating structure between individuals and their bodies by sending a powerful message to society: only a determined physical stereotype of beauty is valued. Reiterated by other primary agents of socialisation, such as families, peers and schools, the idea is taken seriously mainly by young women. "Thus, it can be argued that the mass media not only reinforces cultural stereotypes but also strengthens gender behaviour patterns".

${ }^{17}$ Continua Weiderer (1993) sobre los medios de comunicación: “... Ein bedeutender Aspekt soziåaler Realität, der vom Fernsehen vermittelt wird, ist die Vorstellung über adäquates Geschlechtsrollenverhalten, über gesellschaft- lich akzeptierte Lebensgestaltungen von Frauen und

\begin{tabular}{l|l} 
Futhark I2 (2017) & I5 \\
II-I9 &
\end{tabular}


apuntaba Weiderer (1993): "die Funktion der Medien als Sozialisationsagenten erlangt einen immer höheren Stellenwert, bean- sprucht doch der Medienkonsum einen ständig steigenden Anteil am Leben der Menschen" (Weiderer 1993: 15).

Con una mentalidad de víctima y agresor que poco a poco cambia mediante esa tensión sexual constante entre ambos. Vemos cómo comienza a darse lugar una reacción psicológica en la protagonista, "Erneut schenkt er mir einen von diesen Blicken, unter denen es mir heiß und kalt wird. Wenn er doch nur kein Warrior und ich keine Sklavin wäre!" (Minden 2013: sp) sintiéndose atraída sexualmente por su agresor, con el que tiene un pasado casual y nada sexual, pero que ya había quedado marcada en ella la atracción física por él. Nos plantea una situación que sabemos que puede darse en la realidad. Un síndrome ${ }^{18}$ derivado de la necesidad afectiva de la víctima por parte de su opresor que se da en muchos casos y acaba convirtiéndose en este caso en una necesidad sexual. Esta reacción psicológica conocida como síndrome de Estocolmo, "been used to describe the positive emotional bond a kidnap victim may develop towards their captor. It is speculated that this bond develops as part of the victims defence mechanism to allow them to sympathize with their captor, leading to an acceptance of the situation, limiting defiance / aggression toward the captor and thus maintaining survival in an otherwise potentially high-risk scenario" (De fabrique et al. 2007: sp).

Esta visión de mundo, donde el agresor es visto por el público como un héroe, merecedor del premio, y la víctima como un ser sin derechos, es un entorno muy diferente al que estamos acostumbrados, pero que en realidad

Männern. Da die durch das Fernsehen verbreitete Sichtweise Wirkungen auf die Lebensperspektive der RezipientInnen für sich und für das andere Geschlecht ausübt, erscheint eine genaue Kenntnis der Medienrealität unabdingbar, um gegebenenfalls Mißstände aufzu- decken und auf die Vermittlung adäquater Identifikationsmöglichkeiten für beide Geschlechter hinzuwirken" (Weiderer 1993: I5). Los medios de comunicación, internet etc., nos forman el modelo de belleza ideal en los últimos años, el ideal de belleza de mujer ya no es solo la delgadez. En muchos artistas mediáticos podemos ver que su físico es símbolo de belleza, y sus cuerpos están alejados de esa delgadez clásica. Son cuerpos redondos, de caderas anchas y pechos voluptuosos. Personajes muy puparles en todo el mundo como son la familia Kardashian, Beyoncé, Jennifer López o Niki Minaj, por citar los más famosos y actuales. Actrices, modelos, cantantes, $y$ artistas exponen sus cuerpos "imperfectos" de manera que el atractivo para los demás llega a cambiar estos estereotipos de belleza. No es el ideal tradicional. Malone (2017: 58) en un estudio sobre la influencia de la familia Kardashian: "The Kardashian-Jenners partially encourage the fourth-wave feminist notions of body-positivity and sex-positivity in their target audience, and they have made their target audience trans-aware, and trans- accepting" ...As the results about body-positivity show, the family makes their target audience feel good about their own looks through their words. This is the surface level de nition of body positivity: feeling good about the way you look".

${ }^{18}$ El conocido síndrome lo define también Rosso (2007: 69) como "un estado psicológico en el que la victima... detenida contra su propia voluntad, desarrolla una relación de complicidad con el secuestrador". Este hecho puede darse por diversas causas. El atractivo físico del captor en este caso es lo que hace a la protagonista aceptar en su interior sensaciones contrarias a lo racional. 
representa la masculinidad pues como dice Chávez Mina (2016) “... la masculinidad se atribuye a la racionalidad, la frialdad, la fuerza, el dominio, la dureza de carácter y el poder socialmente aceptado en sociedades donde priman concepciones de subordinación" (Mina 2016: 26).

Hace que veamos una vida alejada de la realidad, o quizás no tanto. Juega con el lenguaje y las situaciones de manera que las necesidades naturales cita... y sexuales se mezclan. Plantea que, para muchos la frontera entre el dolor y el placer no está del todo clara. Es un texto para evadirse, de liberación sexual ${ }^{19}$. Pero también hace preguntarnos dónde está el límite de cada uno y de lo que significa para cada persona esa libertad sexual ${ }^{20}$ y esa misma libertad de fantasear con ella. Dentro del género porno ${ }^{21}$, el papel de la mujer casi siempre es el de ser dominada, en la mayoría de veces por el hombre. Podemos ver pornografía en la que las mujeres fingen ser violadas, el hecho de que las mujeres participen en este tipo de películas dentro del género porno, hace ver una realidad de la que no se habla.

\section{Referencias bibliográficas}

-Bibliografía primaria

Minden, Inka L (20I3), Jax, Warrior Lover. Amazon Media EU.

-Bibliografía secundaria

Bravo, P. C.; Moreno, P. V. (2007). La interiorización de los estereotipos de género en jóvenes y adolescentes". Revista de Investigación Educativa, 25(I), 35-38.

\footnotetext{
19 Por eso cobran importancia textos como este, ya que como apunta Flicker (2008: 125) "die "erste Frauenbewegung" kämpfte bis ins 20. Jahrhundert um grundsätzliche demokratische Rechte wie Wahlrecht, Recht auf Bildung, Arbeitsrecht u. v. m. Die "zweite Frauenbewegung" der 1960/I970er Jahre fokussierte neben einer Systemkritik am institutionalisierten Kapitalismus die Gleichstellung von Män- nern und Frauen im beruflichen wie im privaten Leben”.

${ }^{20}$ Rodríguez y Pérez (2013: 37) hablan sobre los problemas de la libertad sexual para la mujer: "los discursos mediáticos celebran el placer femenino, pero esta celebración se acompaña de nuevos imperativos físicos, mentales y técnicos en el ejercicio de la sexualidad. Estos imperativos paradójicamente limitan la libertad femenina, al mismo tiempo que eximen a la sexualidad de los componentes de la espontaneidad y la creatividad". Por ello es importante que textos como el que se analiza sean visibles y se exponga así una realidad que existe entre las mujeres.

${ }^{21}$ En la actualidad el lugar donde se consume más pornografía es en Internet. La facilidad para crear y añadir contenido a una web hace que haya miles de opciones en cuanto a gustos. De fácil acceso y gratuito, hace que el número de consumidores aumente en gran medida. En este tipo de páginas webs podemos ver como hay gran cantidad de películas donde las mujeres "fingen" ser violadas. Basta con hacer una búsqueda rápido en las páginas webs más visitadas por los usuarios que consumen este tipo de películas pornográficas y eróticas, tales como www.redtube.com, www.pasion.com o www.theporndude.com.
} 
Calvo Shadid, A. (20II). Sobre El Tabú, el tabú Lingüístico y su estado de la Cuestión. Revista Káñina XXXV. En: http://ww w.redalyc.org/articulo.oa?id=442487900 I I (consulta 7/2/20I7).

Castellanos Llanos, G. (2006). Erotismo, violencia y género: deseo femenino, femineidad y masculinidad en la pornografía, 53-65. En: http://bibliotecadigital.univalle.edu.co:8080/bitstream//0893/2637/I/art4.pdf (consulta 7/2/20I7).

De Fabrique, N.; Romano, S. J.; Vecchi, G. M.; Van Hasselt, V. B. (2007). Understanding stockholm sindrome. FBI L. Enforcement Bull 76 (I0). En: htups/heinonline.org/HOL/LandingPage?handle=hein.journals/fbileb76\&div=698id=\&page=(consulta 7/2/2017).

Elster, J. (1990). Racionalidad y normas sociales. Revista Colombiana De Sociología I (I). En: https://revistas.Unal.edu.co/index.php/RECS/article/view/8726 (consulta 7/2/20I7).

Flicker E. (2008). Der Diskurs "Frauenbewegung” in den Medien. En: Dorer J.; Geiger B.; Köpl R. (Hrsg.). Medien - Politik - Geschlecht. Feministische Befunde zur politischen Kommunikationsforschung. En: https://link.springer. com/chapter/I0.1007/978-3-531-91096-3_9 (consulta 7/2/2017).

Hill, A. (2002). Big Brother: the real audience. Television \& New Media 3 (3): $323-$ 340.

James, E. L. (20I I). Fifty shades of Grey. Uk. Vintage.

Llorente, M. E. (2013). Erotismo y pornografía: revisión de enfoques y aproximaciones al concepto de erotismo y de literatura erótica. Anuario de Letras. Lingüística y Filología 40, 359-375.

Maloney, A. R. (2017). The Influence of the Kardashian-Jenners on Fourth Wave Feminism Through Digital Media Platforms. ELON JOURNAL 8 (2). En: htups//unw.elon.edu/ulacademics/communications/journal/wp-content/uploads /sites/153/20I7/I2/Fal20I7Journal.pdffpage-48 (consulta 7/2/2017).

Mendible, M. (2004). Humiliation, subjectivity, and reality TV. Feminist Media Studies 4 (3): 335-338.

Meza Villalba, J.A. (2018), El tabú sexual más allá de la moral. Index Enfermería 17 (2). En: htts//repository.usta.edu.co/handle/l I634/10077 (consulta 7/2/2017).

Mina, C.; Karina, D. (20/6). La representación mediática de la belleza masculina: análisis de contenido de la construcción de la masculinidad en adolescentes en las secciones Vibra y En boga de la revista Generación2 I. Quito: UCE.

Morales, H. (2005). Sociolingüística del tabú. Interlingüística I6: 7-20.

Preciado, B. (2007), "Mujeres en los márgenes". El País, vol. I3. En: http://pmayo bre.webs.uvigo.es/textos/varios/despues_del_feminismo.pdf (consulta 7/ 2/2017).

Rodríguez, T. / Pérez, I. (20I4). "La sexualidad femenina en discursos de la prensa popular y la ficción televisiva”. Comunicación y sociedad, n²1, I5-4I. En: 
htup//hnwwscielo.orgmx/scielo.php?pid=S0188-252X20140001000028script=sd_arttext (consulta 7/2/2017).

Rosso, M. (2007). El Síndrome de Estocolmo. Arte, arquitectura y sociedad digital, 69. En: https://books.google.es/books?hl=es\&lr=\&id=xns7xvOE es8C\&oi=fnd\&pg=PA69\&dq=sindrome+de+estocolmo+definición\&ots=_A OIVBhgID\&sig=4EZO2CNfXbJv8OjEgYyhwVdvKto\#v=onepage\&q\&f=false (consulta 7/2/2017)

Sepúlveda, A. R.; Calado, M. (2012). "Westernization: the role of mass media on body image and eating disorders". En Relevant topics in eating disorders. InTech. En: https://scholar.google.es/scholr?hl=es\&as_sdt=0\%25\&q $=$ Th e+Role+of+Mass+Media+on+Body+Image+and+Eating+Disorders+\&btnG $=($ consulta $7 / 2 / 2017)$

Weiderer, M. (1993). Das Frauen-und Männerbild im deutschen Fernsehen: eine inhaltsanalytische Untersuchung der Programme von ARD, ZDF und RTL plus. Medienpsychologie 4, I5-34. 
